\title{
Optimality Conditions and Scalarization of Approximate Quasi Weak Efficient Solutions for Vector Equilibrium Problem
}

\author{
Yameng Zhang, Guolin Yu $\mathbb{D}$, and Wenyan Han \\ Institute of Applied Mathematics, North Minzu University, Yinchuan 750021, China \\ Correspondence should be addressed to Guolin Yu; guolin_yu@126.com
}

Received 13 May 2020; Revised 6 July 2020; Accepted 30 August 2020; Published 15 September 2020

Academic Editor: Shuping He

Copyright $(2020$ Yameng Zhang et al. This is an open access article distributed under the Creative Commons Attribution License, which permits unrestricted use, distribution, and reproduction in any medium, provided the original work is properly cited.

\begin{abstract}
This paper is devoted to the investigation of optimality conditions for approximate quasi weak efficient solutions for a class of vector equilibrium problem (VEP). First, a necessary optimality condition for approximate quasi weak efficient solutions to VEP is established by utilizing the separation theorem with respect to the quasirelative interior of convex sets and the properties of the Clarke subdifferential. Second, the concept of approximate pseudoconvex function is introduced and its existence is verified by a concrete example. Under the assumption of introduced convexity, a sufficient optimality condition for VEP in sense of approximate quasi weak efficiency is also presented. Finally, by using Tammer's function and the directed distance function, the scalarization theorems of the approximate quasi weak efficient solutions of the VEP are proposed.
\end{abstract}

\section{Introduction}

Vector equilibrium, which is closely related to complementarity problems, variational inequalities, and fixed point theory, is one of the momentous contents in the field of applied mathematics. The characteristics and optimality conditions of various solutions are the key study of vector equilibrium problems. For instance, the optimality conditions for efficient solutions to vector equilibrium problem were presented in $[1]$; the literatures $[2,3]$ derived the optimality conditions of weakly efficient solutions; some optimality conclusions related to several properly efficient solutions were established in [4-7]. In practical applications, the majority of solutions obtained by numerical algorithms are approximate solutions. Undoubtedly, it is of great theoretical and practical significance to study the approximate solutions of vector equilibrium problem. In recent years, the concept of approximate weak efficient solutions for vector equilibrium problem was introduced and its properties were discussed in $[8,9]$. Das and Nahak $[10]$ presented the concept of approximate quasi weak efficient solutions to vector equilibrium problem and examined its optimality conditions by generalized derivatives. One of the main purposes of this paper is to establish the necessary optimality condition for approximate quasi weak efficient solutions to vector equilibrium problem via the quasirelative interior-type separation theorem of convex sets. It is worth mentioning that our method is different from that of Das and Nahak [10].

Convexity and its generalization play a critical role in optimization and vector equilibrium theory, especially in establishing the sufficient optimality conditions. For instance, Gong [11, 12] derived the sufficient optimality condition to approximate efficient solutions for vector equilibrium problem under the cone convexity; under the assumptions of arcwise connected functions, the sufficient optimality conditions with regard to properly efficient solutions to vector equilibrium problem are presented in the literature [13]; based on the assumption of generalized cone subconvexlikeness, the literature [14] proposed the properties of globally efficient solutions to vector equilibrium problem. In this paper, we will introduce notion of approximate quasi-pseudoconvex function in terms of Clarke subdifferential, and under its assumption, we establish the sufficient optimality condition of approximate quasi weak efficient solutions to vector equilibrium problem, which is another aim of this paper. 
Scalarization is to transform a vector problem into a numerical (scalar) problem which is equivalent to primal vector problem under mild conditions. There is no doubt that scalarization is one of the core topics in the study of vector equilibrium problem. In present paper, we will utilize Tammer's nonlinear scalar function and the directed distance function to deal with the scalarization theorems for the approximate quasi weak efficient solutions to vector equilibrium problem.

In the view of the above discussion, the paper will examine the optimality conditions and scalarization theorems in sense of approximate quasi weak efficient solutions to vector equilibrium problem. The article is arranged as follows: in section 2, some symbols, concepts, and lemmas will be presented, which will be used in the subsequent sections; Section 3 is devoted to establish the optimality conditions for approximate quasi weak efficient solutions to the discussed vector equilibrium problem; in section 4 , the scalarization theorems will be proven.

\section{Preliminaries}

Throughout the paper, we set

$$
\mathbb{R}_{+}^{n}=\left\{\left(x_{1}, x_{2}, \ldots, x_{n}\right): x_{i} \geq 0, \quad i=1,2, \ldots, n\right\} .
$$

Let $X$ and $Y$ be real Banach spaces with topological dual spaces $X^{*}$ and $Y^{*}$, respectively, and $\mathbb{B}(\bar{x}, r)$ stands for the open ball of radius $r>0$ around $\bar{x} \in X$. For all $x \in X$ and $x^{*} \in X^{*}$, the value of linear functional $x^{*}$ at $x$ be denoted by $\left\langle x^{*}, x\right\rangle$. Let $Q$ be a pointed closed convex cone in $Y$, then the dual cone of $Q$ be defined as (see [15])

$$
Q^{*}=\left\{y^{*} \in Y^{*}:\left\langle y^{*}, y\right\rangle \geq 0, \quad \forall y \in Q\right\} .
$$

Without other specifications, we always suppose that $Q$ is a pointed closed convex cone in $Y$. We will use the following properties of $Q$.

Lemma 1 (see [16]). If $y^{*} \in Q^{*} /\{0\}$ and $y \in$ intQ, then $\left\langle y^{*}, y\right\rangle>0$, where int represents the interior of a set.

Let $K$ be a nonempty subset of $X$, and the Clarke contingent cone (see [1]) to set $K$ at point $\bar{x} \in K$ is defined as $T(\bar{x} ; K)=\left\{y \in X: \exists t_{n} \longrightarrow 0, y_{n} \longrightarrow y, \quad\right.$ s.t $\left.\bar{x}+t_{n} y_{n} \in K\right\}$.
The Clarke normal cone (see [1]) associated with $T(\bar{x} ; K)$ is denoted by

$$
N(\bar{x} ; K)=\left\{\xi \in X^{*}:\langle\xi, y\rangle \leq 0, \quad \forall y \in T(\bar{x} ; K)\right\} .
$$

Especially when $K$ be a convex set, the Clarke contingent cone to set $K$ at $\bar{x}$ is given by (see [15])

$$
T(\bar{x} ; K)=\operatorname{cl}\{y \in X: y=\beta(x-\bar{x}), \quad x \in K, \beta>0\} .
$$

The Clarke normal cone to set $K$ at $\bar{x}$ is

$$
N(\bar{x} ; K)=\left\{\xi \in X^{*}:\langle\xi, x-\bar{x}\rangle \leq 0, \quad \forall x \in K\right\},
$$

where $\mathrm{cl}$ stands for the closure of a set.

Let $F: X \longrightarrow Y$ be a mapping. $F$ is said to be locally Lipschitz at $\bar{x} \in X$, if there exist constant $L>0$ and $r>0$ such that

$$
\left\|F\left(x_{1}\right)-F\left(x_{2}\right)\right\| \leq L\left\|x_{1}-x_{2}\right\|, \quad \forall x_{1}, x_{2} \in \mathbb{B}(\bar{x}, r) .
$$

If for any $x \in X, F$ is locally Lipschitz at $x$, then $F$ is called locally Lipschitz mapping. In particular, for a realvalued locally Lipschitz function $f: X \longrightarrow \mathbb{R}(\mathbb{R}$ denotes real number), the Clarke generalized directional derivative of $f$ at $\bar{x} \in X$ in the direction $d \in X$ is given by (see [15])

$$
\begin{array}{r}
f^{\circ}(\bar{x} ; d)=\lim _{y \longrightarrow \bar{x}} \sup _{\lambda \longrightarrow 0^{+}} \frac{f(y+\lambda d)-f(y)}{\lambda}, \\
\partial f(\bar{x})=\left\{\xi \in X^{*}: f^{\circ}(\bar{x} ; d) \geq\langle\xi, d\rangle, \quad \forall d \in X\right\},
\end{array}
$$

which is defined as the Clarke subdifferential of $f$ at $\bar{x}$.

We present below some significant properties of locally Lipschitz function that we shall use in the sequel.

Lemma 2 (see $[15,17]$ ). Let function $f: K \subset X \longrightarrow \mathbb{R}$ is locally Lipschitz at $\bar{x} \in K$, if $\bar{x}$ is the minimum value point of $f$ on $K$, then

$$
0 \in \partial f(\bar{x})+N(\bar{x} ; K)
$$

Lemma 3 (see [15]). Let $f_{i}: X \longrightarrow \mathbb{R}, i=1, \ldots, m$, be locally Lipschitz at $\bar{x} \in X$, then function $\varphi(\cdot)$ : $=\max \left\{f_{i}(\cdot): i=1, \ldots, m\right\}$ is also locally Lipschitz at $\bar{x}$, and

$$
\begin{aligned}
\partial \varphi(\bar{x}) \subset & \bigcup\left\{\sum_{i=1}^{m} \lambda_{i} \partial f_{i}(\bar{x}): \lambda_{i} \geq 0, \quad i=1,2, \ldots m, \sum_{i=1}^{m} \lambda_{i}=1, \quad \lambda_{i}\left(f_{i}(\bar{x})-\varphi(\bar{x})\right)=0\right\}, \\
& \partial\left(f_{1}+\cdots+f_{m}\right)(\bar{x}) \subset \partial f_{1}(\bar{x})+\cdots+\partial f_{m}(\bar{x}) .
\end{aligned}
$$

Let $K \subset X$ be a nonempty subset, and $F: K \times K \longrightarrow Y$ be a mapping. Consider the following vector equilibrium problem (VEP):
(VEP) find $\bar{x} \in K$, such that $F(\bar{x}, x) \notin-Q \backslash\{0\}, \quad \forall x \in K$. 
Given $\bar{x} \in K, F_{\bar{x}}: K \longrightarrow Y$ be vector-valued mapping of one variable, which is defined by

$$
F_{\bar{x}}(x):=F(\bar{x}, x), \quad \forall x \in K .
$$

Throughout this paper, it is always assumed that $F_{\bar{x}}(\bar{x})=$ 0 and

$$
F_{\bar{x}}(K)=F(\bar{x}, K)=\cup_{x \in K} F(\bar{x}, x)
$$

Definition 1 (see [10]). Let $K \subset X$ be a nonempty subset, $\varepsilon \geq 0, e \in \operatorname{int} Q . \bar{x} \in K$ is called an $\varepsilon e$-quasi weak efficient solution to VEP, if

$$
F(\bar{x}, x)+\varepsilon\|x-\bar{x}\| e \notin-\operatorname{int} Q, \quad \forall x \in K .
$$

The notion of $\varepsilon e$-quasi weak efficient solution is illustrated by the following example.

Example 1. Let $X=Y=\mathbb{R}, K=\mathbb{R}_{+}, Q=\mathbb{R}_{+}^{2}$, and $\bar{x} \in K$. Consider the following questions:

$$
F(\bar{x}, x)=(-|x-\bar{x}|,|x-\bar{x}-| x-\bar{x} \mid), \quad \forall x \in K .
$$

Taking $\varepsilon=1$ and $e=(1,1)$, then

$$
\begin{aligned}
F(\bar{x}, x)+\varepsilon\|x-\bar{x}\| e= & (\dashv x-\bar{x}|,| x-\bar{x}-|x-\bar{x}|) \\
& +(|x-\bar{x}|,|x-\bar{x}|) \\
= & (0, x-\bar{x}) .
\end{aligned}
$$

Taking $\bar{x}=0$, for all $x \in K$, we obtain

$$
F(\bar{x}, x)+\varepsilon\|x-\bar{x}\| e=(0, x) \notin-\text { intQ. }
$$

Hence, 0 is an $\varepsilon$-quasi weak efficient solution of VEP.

It is well known that, for a nonempty convex set, its interior may be empty, but its quasirelative interior is always nonempty (see [18]). In this paper, we will prove the optimality condition of VEP by the separation theorem with respect to the quasirelative interior of convex sets (see [19]).

Definition 2 (see [18]). Let $K \subset X$ is a convex subset; the quasirelative interior of $K$ denoted by qri $K$ is defined as

$$
\operatorname{qri} K=\{x \in K: \text { clcone }(K-x) \text { is a linear subset of } X\} \text {, }
$$

where $\mathrm{cl}$ and cone stand for closure and cone hull.

Lemma 4 (see [19]). Let $M$ and $N$ be nonempty convex subsets of $Y$, qriM $\neq \varnothing$ and $q$ ri $N \neq \varnothing$, and clcone (qriM - qriN) is not a linear subset of $Y$, then there exists $\lambda \in Y^{*}\{0\}$ such that

$$
\langle\lambda, m\rangle \leq\langle\lambda, n\rangle, \quad \forall m \in M, \forall n \in N .
$$

\section{Optimality Conditions}

In this section, first, we propose a necessary optimality condition for $\varepsilon e$-quasi weak efficient solutions to VEP by using separation theorem in terms of quasirelative interiors of a convex set. Second, the concept of approximate quasi- pseudoconvex function is introduced and a sufficient optimality conditions is established under the introduced generalized convexity. Throughout this section, let $K \subset X$ be a nonempty convex set.

Theorem 1. In VEP, let $\bar{x} \in K, \varepsilon \geq 0$, and $e \in$ intQ. Assume that $\bar{x}$ be an ee-quasi weak efficient solution of VEP and $F_{\bar{x}}: X \longrightarrow Y$ is locally Lipschitz mapping at $\bar{x}$. In addition, $\operatorname{qriF}_{\bar{x}}(K) \neq \varnothing$ and clcone $\left[\operatorname{qri}\left(\operatorname{coF}_{\bar{x}}(K)\right)+\right.$ qriQ] is not a linear subspace of $Y$. Then, there exist $\lambda \in Q^{*}\{0\}$ such that

$$
0 \in \partial\left(\lambda \circ F_{\bar{x}}(\bar{x})\right)+N(\bar{x} ; K)+\langle\lambda, e\rangle \varepsilon \mathbb{B},
$$

where co $(\cdot)$ stands for the convex hull, $\mathbb{B}:=\mathbb{B}(0,1)$, and $\lambda{ }^{\circ} F_{\bar{x}}(\cdot):=\left\langle\lambda, F_{\bar{x}}(\cdot)\right\rangle$.

Proof 1. Since

$$
\operatorname{qri} Q=-\operatorname{qri}(-Q),
$$

and clcone $\left[\mathrm{qri}\left(\operatorname{co} F_{\bar{x}}(K)\right)+\mathrm{qriQ}\right]$ is not a linear subspace of $Y$, then clcone $\left[\operatorname{qri}\left(\operatorname{coF}_{\bar{x}}(K)\right)-\operatorname{qri}(-Q)\right]$ is not a linear subspace of $Y$. Moreover,

$$
\operatorname{qri} F_{\bar{x}}(K) \neq \varnothing .
$$

Thus,

$$
\operatorname{qri}\left[\operatorname{co} F_{\bar{x}}(K)\right] \neq \varnothing
$$

Noticing that $\mathrm{qri} Q \neq \varnothing$, it follows from Lemma 4 that there exists $\lambda \in Y^{*} \backslash\{0\}$ such that

$$
\langle\lambda, q\rangle \leq\langle\lambda, x\rangle, \quad \forall q \in-Q, \forall x \in \operatorname{co} F_{\bar{x}}(K),
$$

which means

$$
\langle\lambda, q\rangle \leq\left\langle\lambda, F_{\bar{x}}(x)\right\rangle, \quad \forall q \in-Q, \forall x \in K .
$$

Taking $x=\bar{x}$ in the above formula, we obtain

$$
\langle\lambda, q\rangle \leq 0, \quad \forall q \in-Q .
$$

Hence, $\lambda \in Q^{*} \backslash\{0\}$. Since

$$
F(\bar{x}, x)+\varepsilon\|x-\bar{x}\| e \notin-\text { intQ }, \quad \forall x \in K,
$$

and $F(\bar{x}, \bar{x})=0$, it leads to

$$
F(\bar{x}, x)-F(\bar{x}, \bar{x})+\varepsilon\|x-\bar{x}\| e \notin-\operatorname{int} Q, \quad \forall x \in K .
$$

It follows from $\lambda \in Q^{*} \backslash\{0\}$ and equation (28) that

$$
\langle\lambda, F(\bar{x}, x)-F(\bar{x}, \bar{x})+\varepsilon\|x-\bar{x}\| e\rangle \geq 0, \quad \forall x \in K,
$$

that is

$$
\left\langle\lambda, F_{\bar{x}}(x)\right\rangle-\left\langle\lambda, F_{\bar{x}}(\bar{x})\right\rangle+\langle\lambda, e\rangle \varepsilon\|x-\bar{x}\| \geq 0, \quad \forall x \in K .
$$

On the other hand, let $f:=\lambda \circ F_{\bar{x}}$. Since $F_{\bar{x}}$ is locally Lipschitz at $\bar{x}$, it is obvious that $f$ is a locally Lipschitz function at $\bar{x}$. We set

$$
\varphi(x)=f(x)-f(\bar{x})+\langle\lambda, e\rangle \varepsilon\|x-\bar{x}\|, \quad \forall x \in K .
$$

It follows from equation (29) that 


$$
\varphi(x) \geq 0=\varphi(\bar{x}), \quad \forall x \in K,
$$

which shows that $\bar{x}$ is the minimum point of $\varphi(x)$ on $K$. Taking account of Lemma 2, we arrive at

$$
0 \in \partial \varphi(\bar{x})+N(\bar{x} ; K) .
$$

Since $f$ is a locally Lipschitz function at $\bar{x}$, by Lemma 3 , we have

$$
\begin{aligned}
\partial \varphi(\bar{x}) & \subset \partial(f+\langle\lambda, e\rangle \varepsilon\|\cdot-\bar{x}\|)(\bar{x}), \\
& \subset \partial\left(\lambda \circ F_{\bar{x}}(\bar{x})\right)+\langle\lambda, e\rangle \varepsilon \mathbb{B} .
\end{aligned}
$$

Together with equation (33), we obtain

$$
0 \in \partial\left(\lambda \circ F_{\bar{x}}(\bar{x})\right)+N(\bar{x} ; K)+\langle\lambda, e\rangle \varepsilon \mathbb{B} .
$$

Next, we introduce the concept of approximate quasipseudoconvex function, and under the assumption of this generalized convexity, a sufficient optimality condition for $\varepsilon e$-quasi weak efficient solutions to VEP is derived.

Definition 3. Let $\varepsilon \geq 0$ and the function $f: X \longrightarrow \mathbb{R}$ be locally Lipschitz at $\bar{x} \in X . f$ is said to be $\varepsilon$-quasi-pseudoconvex at $\bar{x}$, if there exists $\xi \in \partial f(\bar{x})$ such that for each $x \in X$ satisfying

$$
\langle\xi, x-\bar{x}\rangle+\varepsilon\|x-\bar{x}\| \geq 0 \Longrightarrow f(x)-f(\bar{x})+\varepsilon\|x-\bar{x}\| \geq 0 .
$$

Example 2. Let $X=\mathbb{R}$, then $f: \mathbb{R} \longrightarrow \mathbb{R}$ is defined by

$$
f(x)= \begin{cases}\frac{2}{3} x^{2}+x, & \text { if } x<0 \\ \ln (x+1), & \text { if } x \geq 0 .\end{cases}
$$

Taking $\varepsilon=1$ and $\bar{x}=0$, by a simple computation, we derive $\partial f(\bar{x})=\{1\}$. For any $x \in \mathbb{R}, 1=\xi \in \partial f(0)$, if

$$
\langle\xi, x-\bar{x}\rangle+\varepsilon\|x-\bar{x}\|=1 \cdot x+1 \cdot|x| \geq 0,
$$

then

$$
f(x)-f(\bar{x})+\varepsilon\|x-\bar{x}\|=f(x)+1 \cdot|x|= \begin{cases}\frac{2}{3} x^{2} \geq 0, & x<0, \\ \ln (x+1)+x \geq 0, & x \geq 0 .\end{cases}
$$

Thus, $f$ is a 1-quasi-pseudoconvex at 0 .

Theorem 2. In VEP, let $\varepsilon \geq 0, e \in \operatorname{int} Q, \bar{x} \in K$, and $F_{\bar{x}}: K \longrightarrow Y$ be locally Lipschitz at $\bar{x}$. Suppose that there exists $\lambda \in Q^{*} \backslash\{0\}$ such that

$$
0 \in \partial\left(\lambda \circ F_{\bar{x}}(\bar{x})\right)+N(\bar{x} ; K)+\langle\lambda, e\rangle \varepsilon \mathbb{B} .
$$

If $\lambda \circ F_{\bar{x}}: K \longrightarrow \mathbb{R}$ is $\langle\lambda, e\rangle \mathcal{E}$-quasi-pseudoconvex at $\bar{x}$, then $\bar{x}$ is $\varepsilon$-quasi weak efficient solutions of VEP.

Proof 2. It follows from (40) that there exist $\xi \in \partial$ $\left(\lambda \circ F_{\bar{x}}(\bar{x})\right), \sigma \in N(\bar{x} ; K)$, and $b \in \mathbb{B}$ such that

$$
\xi+\sigma+\langle\lambda, e\rangle \varepsilon b=0,
$$

which implies for each $x \in K$,

$$
\langle\xi+\sigma+\langle\lambda, e\rangle \varepsilon b, x-\bar{x}\rangle=0,
$$

which is equivalent to

$$
\langle\xi, x-\bar{x}\rangle+\langle\sigma, x-\bar{x}\rangle+\langle\lambda, e\rangle \varepsilon\langle b, x-\bar{x}\rangle=0, \quad \forall x \in K .
$$

Since $K$ is a convex set, according to the definition of contingent cone to set $K$ at $\bar{x}$,

$$
T(\bar{x} ; K)=\operatorname{cl}\{y \in X: y=\beta(x-\bar{x}), \quad x \in K, \beta>0\} .
$$

Therefore,

$$
\langle\sigma, x-\bar{x}\rangle \leq 0, \quad \forall x \in K .
$$

Combining (43) and (44), it is not difficult to find

$$
\langle\xi, x-\bar{x}\rangle+\langle\lambda, e\rangle \varepsilon\langle b, x-\bar{x}\rangle \geq 0, \quad \forall x \in K .
$$

Because $b \in \mathbb{B}$, we obtain $\|b\|=1$. Hence,

$$
\langle b, x-\bar{x}\rangle \leq\|x-\bar{x}\|, \quad \forall x \in K .
$$

Together with equation (46), it leads to

$$
\langle\xi, x-\bar{x}\rangle+\langle\lambda, e\rangle \varepsilon\|x-\bar{x}\| \geq 0, \quad \forall x \in K .
$$

Since $\lambda \circ F_{\bar{x}}$ is $\langle\lambda, e\rangle \mathcal{E}$-quasi-pseudoconvex at $\bar{x}$, by Definition 3, we obtain

$$
\lambda \circ F_{\bar{x}}(x)-\lambda \circ F_{\bar{x}}(\bar{x})+\langle\lambda, e\rangle \varepsilon\|x-\bar{x}\| \geq 0, \quad \forall x \in K .
$$

In view of $F_{\bar{x}}(\bar{x})=0$, we arrive at

$$
\lambda \circ F_{\bar{x}}(x)+\langle\lambda, e\rangle \varepsilon\|x-\bar{x}\| \geq 0, \quad \forall x \in K .
$$

Suppose that $\bar{x}$ is not $\varepsilon e$-quasi weak efficient solutions of VEP, then there exists $\widehat{x} \in K$ such that

$$
F(\bar{x}, \widehat{x})+\varepsilon\|\widehat{x}-\bar{x}\| e \in-\operatorname{int} Q .
$$

Since $\lambda \in Q^{*} \backslash\{0\}$, it yields from Lemma 1 that

$$
\langle\lambda, F(\bar{x}, \widehat{x})+\varepsilon\|\widehat{x}-\bar{x}\| e\rangle<0,
$$

which means

$$
\langle\lambda, F(\bar{x}, \widehat{x})\rangle+\langle\lambda, \varepsilon\|\widehat{x}-\bar{x}\| e\rangle<0 .
$$

That is,

$$
\lambda \circ F_{\bar{x}}(\widehat{x})+\langle\lambda, e\rangle \varepsilon\|\widehat{x}-\bar{x}\|<0,
$$

which contradicts (50). Hence, $\bar{x}$ is $\varepsilon e$-quasi weak efficient solutions of VEP.

\section{Scalarization}

In this section, the scalarization theorems for approximate quasi weak efficient solutions to VEP are established by using Tammer's function and the directed distance function, respectively. 


\subsection{Scalarization via Tammer's Function}

Lemma 5 (see [20]). Let $Q \subset Y$ is a pointed closed convex cone and $e \in \operatorname{int} Q \neq \varnothing$ is a fixed element, then Tammer's function $\Psi_{e}^{Q}: Y \longrightarrow \mathbb{R}(\mathbb{R}$ represents the set of real number $)$ is defined by

$$
\Psi_{e}^{Q}(y)=\inf \{t \in R: y \in t e-Q\}, \quad y \in Y .
$$

Then, $\Psi_{e}^{Q}$ is continuous sublinear functional and

$$
\left\{y \in Y: \Psi_{e}^{Q}(y)<0\right\}=-\operatorname{int} Q \text {. }
$$

Definition 4. Let $K$ be a nonempty subset of $X, \varepsilon \geq 0$, and $f: X \longrightarrow \mathbb{R}$ is a real-valued function. Define optimization problem $(P)$ as follows:

$$
(P) \inf f(x), \quad \text { s.t. } x \in K
$$

$\bar{x}$ is called a $\mathcal{E}$-quasi-optimality solution of $(P)$ if

$$
f(x)-f(\bar{x})+\varepsilon\|x-\bar{x}\| \geq 0, \quad \forall x \in K .
$$

Let $\bar{x} \in K$ and $e \in$ intQ. Based on VEP and Tammer's function $\Psi_{e}^{Q}$, consider the following scalarization problem $\left(P_{\Psi_{e}^{Q}}\right)$ :

$$
\left(P_{\Psi_{e}^{Q}}\right) \inf \Psi_{e}^{Q}\left(F_{\bar{x}}(x)\right), \quad \text { s.t. } x \in K \text {. }
$$

Theorem 3. Let $\varepsilon \geq 0$ and $e \in \operatorname{int} Q$. If $\bar{x} \in K$ is $\varepsilon$-quasi weak efficient solutions of VEP, then $\bar{x}$ is $\varepsilon$-quasi-optimality solutions of scalarization problem $\left(P_{\Psi_{e}^{Q}}\right)$.

Proof 3. Since $\bar{x} \in K$ is $\varepsilon$-quasi weak efficient solutions of VEP, then

$$
F(\bar{x}, x)+\varepsilon\|x-\bar{x}\| e \notin-\operatorname{int} Q, \quad \forall x \in K .
$$
$\Psi_{e}^{Q}$,

Considering Tammer's nonlinear scalarization function

$$
\Psi_{e}^{Q}(y)=\inf \{t \in \mathbb{R}: y \in t e-Q\}, \quad y \in Y .
$$

According to Lemma 5 and combining (60) and (61) yield that

$$
\Psi_{e}^{Q}(F(\bar{x}, x)+\varepsilon\|x-\bar{x}\| e) \geq 0, \quad \forall x \in K .
$$

Since $\Psi_{e}^{Q}$ is continuous sublinear functional, it holds that

$$
\Psi_{e}^{Q}(F(\bar{x}, x))+\varepsilon\|x-\bar{x}\| \geq 0, \quad \forall x \in K .
$$

Since $F(\bar{x}, \bar{x})=0$, then

$$
\Psi_{e}^{Q}(F(\bar{x}, x))-\Psi_{e}^{Q}(F(\bar{x}, \bar{x}))+\varepsilon\|x-\bar{x}\| \geq 0, \quad \forall x \in K .
$$

Therefore, $\bar{x}$ is $\varepsilon$-quasi-optimality solutions of scalarization problem $\left(P_{\Psi^{Q}}\right)$.

Theorem 4. Let $\varepsilon \geq 0, e \in \operatorname{int} Q$, and $\bar{x} \in K$. Suppose function $\varphi: Y \longrightarrow \mathbb{R}$ satisfying the following: (i) $\varphi$ is monotone with respect to the pointed closed convex cone $Q$, that is, if $y_{1}-y_{2} \in Q$, then $\varphi\left(y_{1}\right) \geq \varphi\left(y_{2}\right)$

(ii) $\varphi(0)=0$, and $\varphi$ is positively homogeneous functional that means $\varphi(a y)=a \varphi(y), a>0$

(iii) $\varphi(-\varepsilon e)<-\varepsilon$

Let scalarization problem $\left(P_{\varphi}\right)$ be defined by

$$
\left(P_{\varphi}\right) \inf \varphi\left(F_{\bar{x}}(x)\right), \quad \text { s.t. } x \in K \text {. }
$$

If $\bar{x}$ is $\varepsilon$-quasi-optimality solutions of scalarization problem $\left(P_{\varphi}\right)$, then $\bar{x}$ is $\varepsilon$-quasi weak efficient solutions of VEP.

Proof 4. If $\bar{x}$ is not $\varepsilon e$-quasi weak efficient solutions of VEP, there would exist $\hat{x} \in K$ such that

$$
F(\bar{x}, \widehat{x})+\varepsilon\|\widehat{x}-\bar{x}\| e \in-\operatorname{int} Q,
$$

which is equivalent to

$$
-\varepsilon\|\widehat{x}-\bar{x}\| e-F(\bar{x}, \widehat{x}) \in \operatorname{int} Q .
$$

Then, we have

$$
-\varepsilon\|\hat{x}-\bar{x}\| e-F(\bar{x}, \hat{x}) \in Q
$$

Since $\varphi$ is monotone with respect to $Q$,

$$
\varphi(F(\bar{x}, \widehat{x})) \leq \varphi(-\varepsilon\|\widehat{x}-\bar{x}\| e) .
$$

Noticing that $\bar{x}$ is $\varepsilon$-quasi-optimality solutions of problem $\left(P_{\varphi}\right)$, we obtain

$$
\varphi(F(\bar{x}, \widehat{x}))-\varphi(F(\bar{x}, \bar{x}))+\varepsilon\|\widehat{x}-\bar{x}\| \geq 0 .
$$

Because $F(\bar{x}, \bar{x})=0$ and $\varphi(0)=0$, it holds that

$$
\varphi(F(\bar{x}, \widehat{x}))+\varepsilon\|\widehat{x}-\bar{x}\| \geq 0 .
$$

Combining (69) and (71), we obtain

$$
\varphi(-\varepsilon\|\widehat{x}-\bar{x}\| e) \geq-\varepsilon\|\widehat{x}-\bar{x}\| .
$$

Since $\varphi$ is positively homogeneous functional,

$$
\varphi(-\varepsilon e) \geq-\varepsilon,
$$

which contradicts to condition (iii).

4.2. Scalarization via the Directed Distance Function. Let us introduce the concept of directed distance function.

Definition 5 (see [21]). Let $A \subseteq Y$ is a nonempty subset, then the directed distance function $\Delta_{A}: Y \longrightarrow \mathbb{R}$ be defined as

$$
\Delta_{A}(y)=d_{A}(y)-d_{Y \backslash A}(y), \quad y \in Y,
$$

where

$$
d_{A}(y)=\inf _{x \in A}\|y-x\| .
$$


Lemma 6 (see [21]). Let $A \subseteq Y$ is a nonempty subset, then the following properties hold:

(i) $\Delta_{A}$ is real-valued Lipschitz function of rank 1 . (ii) If $A$ is closed, then $A=\left\{y: \Delta_{A}(y) \leq 0\right\}$; if $A$ is a cone, then $\triangle_{A}$ is positively homogeneous; if $A$ is pointed closed convex cone, then $\Delta_{A}$ is sublinear.

(iii) If $A$ is closed convex cone, then $\Delta_{A}$ is nonincreasing with respect to $A$, that is, if $y_{1}, y_{2} \in Y$, then

$$
y_{1}-y_{2} \in A \Longrightarrow \Delta_{A}\left(y_{1}\right) \leq \Delta_{A}\left(y_{2}\right) ; y_{1}-y_{2} \in \operatorname{int} A \Longrightarrow \Delta_{A}\left(y_{1}\right)<\Delta_{A}\left(y_{2}\right) \text {. }
$$

Let $e \in \operatorname{int} Q$ and $\bar{x} \in K$. Based on VEP and the directed distance function $\Delta_{-Q}$, consider the following scalarization problem $\left(P_{\Delta_{-Q}}\right)$ :

$$
\left(P_{\Delta_{-Q}}\right) \inf \Delta_{-Q}\left(F_{\bar{x}}(x)\right), \quad \text { s.t. } x \in K \text {. }
$$

Theorem 5. Let $\varepsilon>0$, $e \in \operatorname{int} Q$ with $\|e\|=1$, and $\bar{x} \in K$. If $\bar{x}$ is $\varepsilon$-quasi weak efficient solutions to VEP, then for any $x \in K$, $\bar{x}$ is an $\varepsilon$-quasi-optimality solution of scalarization problem $\left(P_{\Delta_{-Q}}\right)$.

Proof 5. By the given conditions, we have

$$
F(\bar{x}, x)+\varepsilon\|x-\bar{x}\| e \notin-\operatorname{int} Q, \quad \forall x \in K .
$$

Since $Q$ is a pointed closed convex cone, by Lemma 6, it yields that

$$
\Delta_{-Q}(F(\bar{x}, x))+\varepsilon\|x-\bar{x}\|_{-Q}(e) \geq \Delta_{-Q}(F(\bar{x}, x)+\|x-\bar{x}\| e) \geq 0, \quad \forall x \in K .
$$

Noticing that $\Delta_{-Q}(e)=\|e\|=1, \quad F(\bar{x}, \bar{x})=0, \quad$ and $\Delta_{-Q}(0)=0$, we arrive at

$$
\Delta_{-Q}(F(\bar{x}, x))-\Delta_{-Q}(F(\bar{x}, x))+\varepsilon\|x-\bar{x}\| \geq 0, \quad \forall x \in K .
$$

Hence, $\bar{x}$ is an $\varepsilon$-quasi-optimality solution of scalarization problem $\left(P_{\Delta_{-}}\right)$.

\section{Conclusions}

Making use of the quasirelative interior-type separation theorem of convex set, we have examined the optimality condition of the approximate quasi weak efficient solutions of VEP. In addition, the scalarization theorems of approximate quasi weak efficient solutions to VEP are also established via using Tammer's function and directed distance function, respectively, and scalarization theorems realize the purpose that solving the approximate quasi weak efficient solutions of vector equilibrium problem is equivalent to solving the approximate quasi-optimality solution of a specific scalar optimization problem.

\section{Abbreviations}

VEP: Vector equilibrium problem.

\section{Data Availability}

No data were used to support the findings of this study.

\section{Conflicts of Interest}

The authors declare that they have no conflicts of interest.

\section{Authors' Contributions}

All authors contributed equally to the manuscript and read and approved the final version of the manuscript.

\section{Acknowledgments}

This research was supported by the Natural Science Foundation of China under Grant no. 11861002, Natural Science Foundation of Ningxia under Grant no. NZ17112, The Key Project of North Minzu University under Grant no. ZDZX201804, and Nonlinear Analysis and Financial Optimization Research Center of North Minzu University.

\section{References}

[1] D. Luu and D. Hang, "Efficient solutions and optimality conditions for vector equilibrium problems," Mathematical Methods of Operations Research, vol. 79, no. 2, pp. 163-177, 2014.

[2] Y. Feng and Q. Qiu, "Optimality conditions for vector equilibrium problems with constraint in Banach spaces," Optimization Letters, vol. 8, no. 6, pp. 1931-1944, 2014.

[3] Z. F. Wei and X. H. Gong, "Kuhn-Tucker optimality conditions for vector equilibrium problems," Journal of Inequalities and Applications, vol. 2010, no. 1, 15 pages, Article ID 842715, 2010.

[4] X. H. Gong, W. T. Fu, and W. Liu, "Super efficiency for avector equilibrium in locally convex topological vectorspaces," in Vector Variational Inequalities and Vector Equilibria: Mathematical Theories, F. Giannessi, Ed., pp. 233-252, Kluwer, Alphen Aan Den Rijn, Netherlands, 2000.

[5] X.-H. Gong, "Optimality conditions for Henig and globally proper efficient solutions with ordering cone has empty 
interior," Journal of Mathematical Analysis and Applications, vol. 307, no. 1, pp. 12-31, 2005.

[6] X.-H. Gong, "Scalarization and optimality conditions for vector equilibrium problems," Nonlinear Analysis: Theory, Methods \& Applications, vol. 73, no. 11, pp. 3598-3612, 2010.

[7] X. J. Long, Y. Q. Huang, and Z. Y. Peng, "Optimality conditions for the Henig efficient solution of vector equilibrium problems with constraints," Optimization Letters, vol. 5, no. 4, pp. 717-728, 2011.

[8] Q. Qiu and X. Yang, "Some properties of approximate solutions for vector optimization problem with set-valued functions," Journal of Global Optimization, vol. 47, no. 1, pp. 1-12, 2010.

[9] Q. Qiu, X. M. Yang, and X. Yang, "Scalarization of approximate solution for vector equilibrium problems," Journal of Industrial \& Management Optimization, vol. 9, no. 1, pp. 143-151, 2013.

[10] K. Das and C. Nahak, "Optimality conditions for approximate quasi efficiency in set-valued equilibrium problems," SeMA Journal, vol. 73, no. 2, pp. 183-199, 2016.

[11] X.-H. Gong, H.-B. Dong, and S.-Y. Wang, "Optimality conditions for proper efficient solutions of vector set-valued optimization," Journal of Mathematical Analysis and Applications, vol. 284, no. 1, pp. 332-350, 2003.

[12] X.-H. Gong, "Optimality conditions for vector equilibrium problems," Journal of Mathematical Analysis and Applications, vol. 342, no. 2, pp. 1455-1466, 2008.

[13] P. Q. Khanh and L. T. Tung, "First and second-order optimality conditions using approximations for vector equilibrium problems with constraints," Journal of Global Optimization, vol. 55, no. 4, pp. 901-920, 2013.

[14] Q. S. Qiu, "Optimality conditions of globally efficient solution for vector equilibrium problems with generalized convexity," Journal of Inequalities and Applications, vol. 2009, no. 1, 13 pages, Article ID 898213, 2009.

[15] F. H. Clarke, Optimization and Nonsmooth Analysis, John Wiley and Sons, New York, NY, USA, 1983.

[16] J. Jahn, Mathematical Vector Optimization in Partially Ordered Linear Spaces, Verlag Peter Lang, Frankfurt, Germany, 1986.

[17] X. J. Long, Y. B. Xiao, and N. J. Huang, “Optimality conditions of approximate solutions for nonsmooth semi-infinite programming problems," Journal of the Operations Research Society of China, vol. 6, no. 2, pp. 1-11, 2017.

[18] J. M. Borwein and A. S. Lewis, "Partially finite convex programming, part I: quasi relative interiors and duality theory," Mathematical Programming, vol. 57, no. 1-3, pp. 15-48, 1992.

[19] F. Cammaroto and B. D. Bella, "Separation theorem based on the quasirelative interior and application to duality theory," Journal of Optimization Theory and Applications, vol. 125, no. 1, pp. 223-229, 2005.

[20] C. Gerth and P. Weidner, "Nonconvex separation theorems and some applications in vector optimization," Journal of Optimization Theory and Applications, vol. 67, no. 2, pp. 297-320, 1990.

[21] A. Zaffaroni, "Degrees of efficiency and degrees of minimality," SIAM Journal on Control and Optimization, vol. 42, no. 3, pp. 1071-1086, 2003. 